\title{
Fronts in high-temperature laminar gas jets
}

\author{
By M. SÁNCHEZ-SANZ $Z^{1}$ A. L. SÁNCHEZ AND A. LIÑÁN ${ }^{2}$ \\ ${ }^{1}$ Area de Mecánica de Fluidos, Universidad Carlos III de Madrid, 28911 Leganés, Spain \\ ${ }^{2}$ ETSI Aeronáuticos, Pl. Cardenal Cisneros 3, 28040 Madrid, Spain
}

(Received 15 July 2005 and in revised form 13 October 2005)

This paper addresses the slender laminar flow resulting from the discharge of a lowMach-number hot gas jet of radius $a$ and moderately large Reynolds number $R_{j}$ into a cold atmosphere of the same gas. We give the boundary-layer solution for plane and round jets with very small values of the ambient-to-jet temperature ratio $\varepsilon$ accounting for the temperature dependence of the viscosity and conductivity typical of real gases. It is seen that the leading-order description of the jet in the limit $\varepsilon \rightarrow 0$ exhibits a front-like structure, including a precisely defined separating boundary at which heat conduction and viscous shear stresses vanish in the first approximation, so that the temperature and axial velocity remain unperturbed outside the jet. Separate analyses are given for the jet discharging into a stagnant atmosphere, when the jet boundary is a conductive front, and for the jet discharging into a coflowing stream, when the jet boundary appears as a contact surface. We provide in particular the numerical description of the jet development region corresponding to axial distances of order $R_{j} a$ for buoyant and non-buoyant jets, as well as the self-similar solutions that emerge both in the near field and in the far field. In all cases considered, comparisons with numerical integrations of the boundary-layer problem for moderately small values of $\varepsilon$ indicate that these front descriptions give excellent predictions for the temperature and velocity fields in the near-axis region.

\section{Introduction}

This paper investigates the discharge of a gas jet at temperature $T_{j}$ into an atmosphere of the same gas at temperature $T_{o} \ll T_{j}$. When the Reynolds number $R_{j}=\rho_{j} U_{j} a / \mu_{j}$, based on the density and viscosity of the jet, $\rho_{j}$ and $\mu_{j}$, the jet velocity $U_{j}$, and the characteristic transverse dimension $a$ (the initial radius for the round jet and the initial half-width for the planar jet), is moderately large the resulting steady flow remains slender and stable. The solution can be described in the boundary-layer approximation with small relative errors of order $R_{j}^{-2}$.

Much of the early work on non-isothermal laminar gas jets is reviewed in Pai (1954). Sufficiently far downstream, the temperature approaches the ambient value, and the solution for the velocity reduces to the well-known self-similar descriptions developed by Schlichting (1933) for the round jet and by Bickley (1937) for the plane jet, while the constant-density solution for the temperature field is due to Yih (1950). The higher-order perturbations to the velocity and temperature fields, associated with small density differences, were calculated by Crane \& Pack (1957) with the assumption of unity Prandtl number, $P r=1$, to facilitate the description of the viscous dissipation effects, which become significant in jets with Mach numbers of order unity. 
A similarly perturbed far-field solution was used in the stability analysis of a plane jet of Kennedy \& Chen (1998).

The solution that appears in the presence of coflow with $\mathrm{Pr}=1$ was investigated by Pai (1949) for the plane jet and by Pai (1952) for the round jet. His work includes analytical results for small temperature and velocity differences between the jet and the coflow, together with sample numerical integrations of the boundary-layer equations for relative temperature differences $\left(T_{j}-T_{o}\right) / T_{o}$ of order unity. Relevant more recent work on the far-field description of a plane jet includes Bansal \& Tack (1978), who developed the similarity solutions for two particular cases: jets with $\operatorname{Pr}=1$ and jets in which the viscosity and heat conductivity are linearly proportional to the temperature.

The self-similar solution associated with a point source of momentum and energy in a gas with vanishing ambient temperature and constant heat conductivity and viscosity was analysed by Bobnev (1982), who later extended his analysis to gases with temperature-dependent transport properties (Bobnev 1985, 1986). Our work also considers jets with temperatures much larger than the ambient, i.e. values of $\varepsilon=T_{o} / T_{j} \ll 1$. Viscous dissipation is not included in the analysis, a simplification that applies when the jet Mach number is sufficiently small. The paper starts by formulating the boundary-layer description of the jet development region of plane and round vertical jets with temperature-dependent heat conductivity and viscosity, including the leading-order front description emerging in the limit $\varepsilon \rightarrow 0$. The jet discharging into a stagnant ambient will be treated first, followed by the jet discharging into a coflow stream. The development considers buoyant and non-buoyant jets, with particular attention given to the distinguished far-field solutions that appear in both cases.

\section{Formulation}

In formulating the problem in dimensionless form, we use as scales those corresponding to the jet development region. Thus, the temperature, density, and viscosity, $T, \rho$ and $\mu$, are scaled with their initial values at the jet exit, while the streamwise and transverse coordinates $x$ and $r$ are scaled with $R_{j} a$ and $a$, respectively, and the axial and radial velocity components $u$ and $v$ are scaled with $U_{j}$ and $\mu_{j} /\left(a \rho_{j}\right)$, leading to a formulation independent of the Reynolds number. The problem reduces to that of integrating

$$
\begin{aligned}
\frac{\partial}{\partial x}(\rho u)+\frac{1}{r^{i}} \frac{\partial}{\partial r}\left(\rho r^{i} v\right) & =0, \\
\rho u \frac{\partial u}{\partial x}+\rho v \frac{\partial u}{\partial r} & =\frac{1}{r^{i}} \frac{\partial}{\partial r}\left(r^{i} \mu \frac{\partial u}{\partial r}\right)+G\left(1-\frac{\varepsilon}{T}\right), \\
\rho u \frac{\partial T}{\partial x}+\rho v \frac{\partial T}{\partial r} & =\frac{1}{\operatorname{Pr}} \frac{1}{r^{i}} \frac{\partial}{\partial r}\left(r^{i} \mu \frac{\partial T}{\partial r}\right),
\end{aligned}
$$

with boundary conditions for $x>0$

$$
\begin{gathered}
r=0: \frac{\partial u}{\partial r}=v=\frac{\partial T}{\partial r}=0, \\
r \rightarrow \infty: u-u_{c}=T-\varepsilon=0,
\end{gathered}
$$

and with initial conditions at $x=0$

$$
\left.\begin{array}{ll}
0 \leqslant r \leqslant 1: & u-1=T-1=0 \\
r>1: & u-u_{c}=T-\varepsilon=0
\end{array}\right\}
$$


The index $i$ takes the value $i=0$ for the planar configuration and $i=1$ for the round jet. Here, $\operatorname{Pr}$ represents the Prandtl number, assumed to be constant in the analysis, $u_{c}$ is the ratio of the coflow velocity to the jet velocity and the dimensionless gravity, $G=\left(g R_{j} a\right) /\left(\varepsilon U_{j}^{2}\right)$, is the inverse squared of the relevant Froude number.

The continuity, momentum and energy conservation equations need to be supplemented with the equation of state written in the near-isobaric approximation, $\rho T=1$, and with the presumed power-law dependence $\mu=T^{\sigma}$ for the temperature variation of the viscosity and heat conductivity, where the exponent, typically ranging from $\sigma=0.5$ to $\sigma=0.75$ for non-ionized gases, will be taken equal to $\sigma=0.70$ in the computations below. Note that the solution satisfies the integral conservation law

$$
\int_{0}^{\infty} 2^{i} r^{i} \rho u(T-\varepsilon) \mathrm{d} r=1-\varepsilon,
$$

obtained by radial integration of (2.3) written in conservative form.

We are concerned here with hot jets, corresponding to small values of the parameter $\varepsilon$. We shall analyse first the case $u_{c}=0$, corresponding to a hot jet discharging into a stagnant cold atmosphere. Although the Prandtl number is less than unity for most gases (e.g. the computations below are for $\mathrm{Pr}=0.70$, corresponding to air), to understand the nature of the front solution that develops it is instructive to consider briefly the case $\operatorname{Pr}=1$. For a non-buoyant jet, one can easily verify that with $\varepsilon=0$ the solution for the velocity reduces to $u=T$ and $v=0$, whereas the temperature is calculated by integration of the nonlinear heat conduction problem

$$
\frac{\partial T}{\partial x}=\frac{1}{r^{i}} \frac{\partial}{\partial r^{i}}\left(r^{i} T^{\sigma} \frac{\partial T}{\partial r}\right)
$$

with initial condition at $x=0$ given by $T=1$ for $0<r<1$ and $T=0$ for $r>1$ and with boundary conditions for $x>0$ given by $\partial T / \partial r=0$ at $r=0$ and $T=0$ as $r \rightarrow \infty$. If the coordinate $x$ represent a dimensionless time, then it is clear from the above that the development of the non-buoyant jet as $x$ increases corresponds to the time evolution of the temperature field in a heated solid with temperature-dependent conductivity and a vanishing outer temperature. The problem was originally considered by Zeldovich $\&$ Kompaneetz (1950), who gave in particular the long-time asymptotic behaviour for $x \gg 1$. They found that, because of its temperature dependence, the heat conductivity vanishes as $T \rightarrow 0$ and heating can only reach a finite distance, thereby yielding a precisely defined boundary $r=r_{f}(x)$ for the resulting hot spot, outside which the temperature remains unperturbed, i.e. $T=0$ for $r \geqslant r_{f}$.

The solution with $\operatorname{Pr}<1$ is qualitatively similar. The exact balance between transverse heat conduction and shear stresses is no longer present in this case, so that $u \neq T$ and $v \neq 0$. The jet temperature and velocity decrease downstream to adjust to their boundary values $u=T=0$. Correspondingly, the radius $r_{f}(x)$ increases to satisfy the condition of constant enthalpy flux

$$
\int_{0}^{r_{f}} 2^{i} r^{i} u \mathrm{~d} r=1
$$

obtained at leading order from (2.7) in the limit $\varepsilon=0$ by imposing a vanishing conductive flux at the boundary. Unlike the case $\operatorname{Pr}=1$, the jet in this case is seen to entrain outer fluid with a positive rate $\Phi_{\infty}(x)=\left(-\rho r^{i} v\right)_{r>r_{f}}$ of order unity to give an increasing jet mass flux $(\mathrm{d} / \mathrm{d} x)\left(\int_{0}^{r_{f}} \rho u r^{i} \mathrm{~d} r\right)=\Phi_{\infty}(x)$. Note that this entrainment rate, which is determined below from the leading-order description, corresponds to radial velocities $v \sim \varepsilon$ for $r>r_{f}$, whereas $v \sim O(1)$ inside the jet. 
The presence of the coflow changes the character of the solution. As before, heat conduction and shear stresses vanish at the jet boundary, although with coflow this is an effect of the increasing density that occurs even in gases with constant heat conductivity and viscosity. The velocity and the temperature remain unperturbed outside, and the jet boundary becomes a fluid surface with $u=u_{c}$ and $T=0$ separating the cold coflow from the low-density gas. Inside the jet, heat conduction and shear stresses progressively adjust the jet temperature and velocity to the boundary values, as the jet radius evolves to accomodate the axial flux of excess enthalpy (2.9). The evolution of the jet boundary $r_{f}(x)$, obtained below from the leading-order description, determines the radial velocity at the bounding fluid surface $v_{f}(x)=u_{c}\left(\mathrm{~d} r_{f} / \mathrm{d} x\right)$, as well as the radial velocity outside the jet, $v=v_{f} r_{f} / r$, which is comparable in magnitude to that found inside the jet.

Separate sections are devoted below to the two distinct cases identified above. To account for the front-like character of the solution associated with the limit $\varepsilon=0$, in integrating (2.1)-(2.3) the boundary conditions (2.5) are replaced with the conditions

$$
T=u-u_{c}=0 \text { and } T^{\sigma} \frac{\partial T}{\partial r}=T^{\sigma} \frac{\partial u}{\partial r}=0 \text { at } r=r_{f} .
$$

The condition of vanishing heat conduction at $r=r_{f}$ can be replaced in the integrations with the condition of constant energy flux given in (2.9), which reveals in particular that accelerating jets, driven by buoyancy or by fast coflow $u_{c}>1$, give $\mathrm{d} r_{f} / \mathrm{d} x<0$, while the opposite is found in buoyancy-free jets with $u_{c} \leqslant 1$.

\section{Hot jet in a stagnant cold atmosphere}

\subsection{Near-field solution}

For $x \ll 1$, mixing is confined to a thin annular mixing layer, not affected by gravity, that can be described in terms of the similarity coordinate $\eta=(r-1) / \sqrt{x}$ by introducing the stream function $\psi=\sqrt{x} F(\eta)$ defined such that $\rho u=F_{\eta}$ and $\rho v=\frac{1}{2}\left(\eta F_{\eta}-F\right) /$ $\sqrt{x}$. The problem reduces to that of integrating

$$
\left[T^{\sigma}\left(T F_{\eta}\right)_{\eta}\right]_{\eta}+F\left(T F_{\eta}\right)_{\eta} / 2=0 \text { and }\left(T^{\sigma} T_{\eta}\right)_{\eta}+P r F T_{\eta} / 2=0 .
$$

In the notation employed here, a coordinate is used as subscript to denote differentiation, e.g. the subscript $\eta$ in the above equations. The axial velocity and the temperature remain unperturbed outside the mixing layer. Furthermore, the condition $v=0$ at $r=0$ requires that $v=0$ inside the jet, so that the mixing layer can only entrain fluid from the ambient, thereby giving $T=F_{\eta}=0$ at $\eta=\eta_{f}$ and $T-1=F-\eta=0$ as $\eta \rightarrow-\infty$ as boundary conditions for (3.1). The above problem was integrated with a shooting scheme, giving the solution shown in figure 1 . The equations were rewritten in terms of the normalized coordinate $\eta / \eta_{f}$ and the integration was started at $\eta / \eta_{f}=1$, with $\eta_{f}$ and $F_{f}=F\left(\eta=\eta_{f}\right)$ entering as parameters in the iterative shooting scheme. The values obtained, $\eta_{f}=2.1625$ and $F_{f}=1.5255$, determine the initial evolution of the jet radius $r_{f}=1+\eta_{f} \sqrt{x}$ as well as the initial value of the jet entrainment $\Phi_{\infty}=-\left(r^{i} \rho v\right)_{r>r_{j}}=F_{f} /(2 \sqrt{x})$. These predictions are compared below in figures 2 and 3 with the numerical results at $x \ll 1$.

\subsection{The development region}

To describe the region of jet development corresponding to distances $x \sim O(1)$, we integrate (2.1)-(2.3) with $\varepsilon=0$. The boundary conditions are those given in (2.4) and (2.10), whereas the solution found at $x \ll 1$ is used to evaluate the initial velocity 


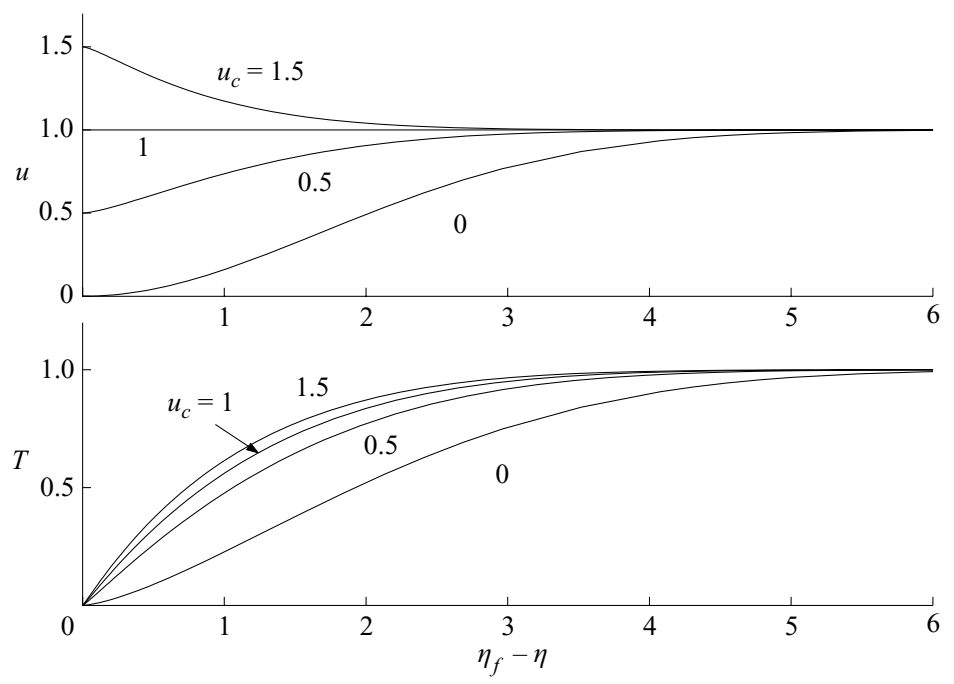

FIGURE 1 . The temperature and velocity profiles across the mixing layer at $x \ll 1$.

and temperature profiles. To simplify the treatment of the free jet boundary, the problem was formulated using the normalized coordinate $R=r / r_{f}(x)$ as a replacement for $r$. To determine the profiles of temperature and velocity, an implicit marching procedure (Anderson, Tannehill \& Pletcher 1984), third-order accurate in $x$ and second-order accurate in $R$, was used to integrate the modified conservation equations in the fixed domain $0 \leqslant R \leqslant 1$, and the integral condition (2.9) was employed to update the value of $r_{f}(x)=\left[1 /\left(\int_{0}^{1} 2^{i} R^{i} u \mathrm{~d} R\right)\right]^{1 /(i+1)}$ at each $x$. A non-uniform grid with minimum spacing $\delta x=6 \times 10^{-5}$ near the entrance and $\delta R=7 \times 10^{-4}$ near the axis was used, and the results were checked to be grid-independent by comparison with results obtained with finer grids in sample computations. For the buoyancy-free jet, the accuracy of the final numerical solution was tested by evaluating the integral form of the momentum equation $\int_{0}^{1} 2^{i} R^{i}\left(u^{2} / T\right) \mathrm{d} R=1$, yielding an error of less than $0.1 \%$.

Profiles of velocity and temperature obtained for the round jet with $G=0$ and $G=5$ are given in figure 2, including the jet boundary $r_{f}(x)$ obtained as part of the integration. The front solution is compared with results of integrations of the original boundary-layer problem (2.1)-(2.6) with two moderately small values of $\varepsilon=(0.02,0.1)$. Both the shape of the profiles and the peak values of temperature and velocity along the axis are accurately described for the range of $x$ considered. The comparison of the peak values $T_{0}=T(r=0, x)$ and $u_{0}=u(r=0, x)$ is given in figure 3, which also exhibits results for the entrainment rate $\Phi_{\infty}(x)=-\left(\rho r^{i} v\right)_{r \rightarrow \infty}$.

\subsection{Far-field solution}

With $G=0$, the jet continues growing as $x$ increases, to approach for $x \gg 1$ a self-similar far-field structure with $r_{f} \gg 1$, which holds as long as the jet remains much hotter than the ambient. The solution must satisfy the condition of constant enthalpy flux, given in (2.9), along with the condition of constant momentum flux $\int_{0}^{r_{f}} 2^{i} r^{i} \rho u^{2} \mathrm{~d} r=1$, which follows from integrating the conservative form of (2.2) with $G=u_{c}=0$. These two conditions, together with the balance between convective and molecular transport, determine the order of magnitude of the jet radius $r_{f} \sim$ $O\left(x^{1 /[2+\sigma(1+i)]}\right)$ and of the temperature and velocity $u \sim T \sim O\left(x^{-(1+i) /[2+\sigma(1+i)]}\right)$. As previously mentioned, the solution corresponding to $\operatorname{Pr}=1$ is that obtained by 


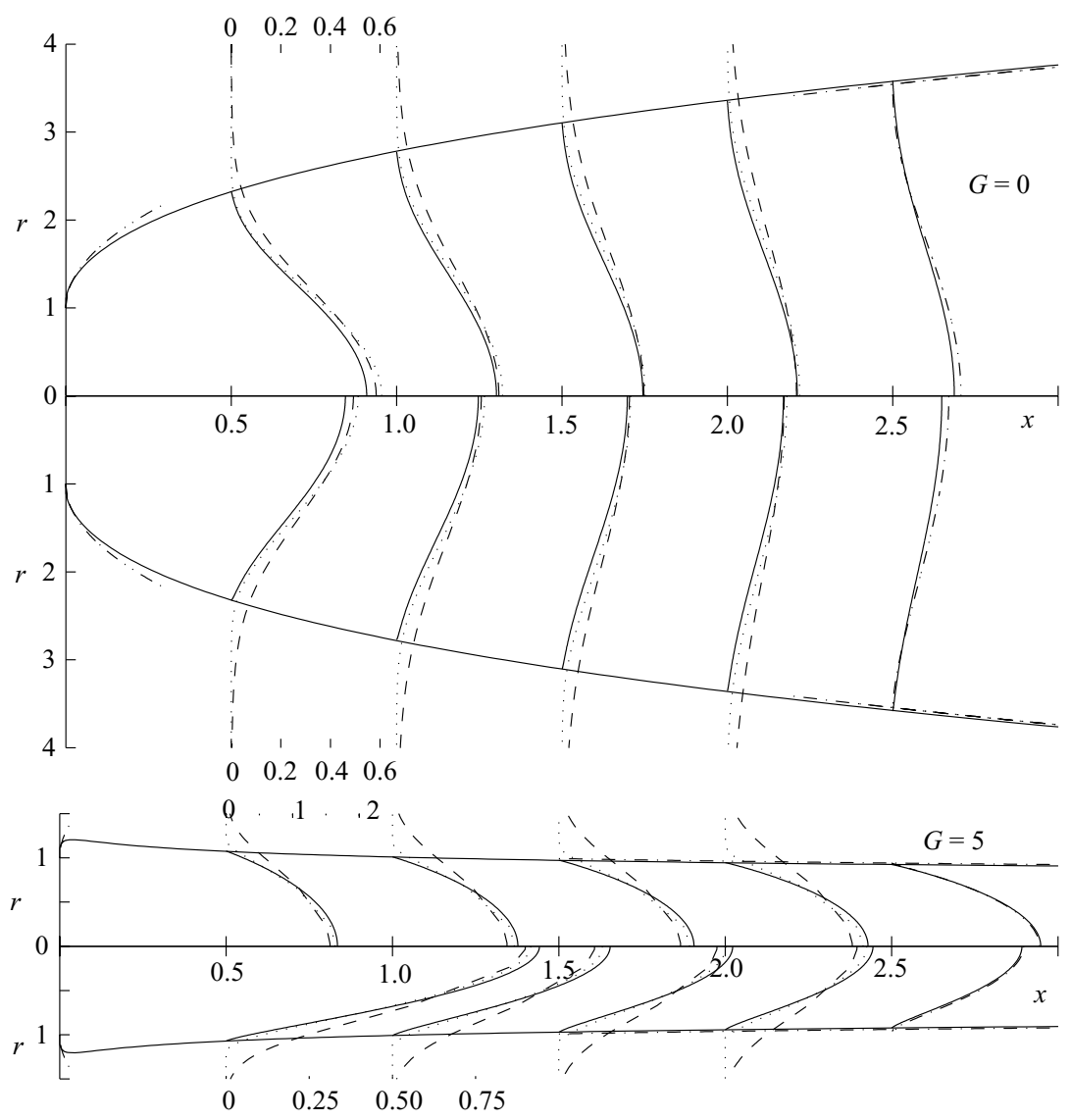

FIGURE 2. The profiles of velocity $u$ (upper half of the plots) and temperature $(T-\varepsilon) /(1-\varepsilon)$ (lower half of the plots) obtained for $\operatorname{Pr}=0.7, \sigma=0.7, u_{c}=0$ and $G=(0,5)$ by integration of (2.1)-(2.3) with $\varepsilon=0$ (solid line), $\varepsilon=0.02$ (dotted lines) and $\varepsilon=0.1$ (dashed lines). The scale for the velocity and temperature is indicated for the profiles at $x=0.5$ on each plot. The profiles at $x=2.5$ are compared with the asymptotic solution for $x \gg 1$ (dot-dashed lines). The jet boundary $r_{f}(x)$ corresponding to the front solution $\varepsilon=0$ is indicated as a solid line for completeness, and the asymptotic predictions for $x \ll 1$ and for $x \gg 1$ are included as dotdashed lines.

Zeldovich \& Kompaneetz (1950) by integrating the nonlinear heat conduction equation (2.8) with boundary conditions $\partial T / \partial r=0$ at $r=0$ and $T=0$ at $r=r_{f}$ and subject to the integral constraint $\int_{0}^{r_{f}} 2^{i} r^{i} T \mathrm{~d} r=1$. On the other hand, the farfield solution for the jet with $\operatorname{Pr} \neq 1$ was originally considered by Bobnev (1985, 1986 ), who reduced the solution to a single second-order nonlinear equation in terms of the parameter $(1-P r) /(\sigma P r)$. The results corresponding to $\operatorname{Pr}=0.7$ and $\sigma=0.7$, not considered specifically in previous works, are given below in terms of the rescaled coordinate $\xi=r / x^{1 /[2+\sigma(1+i)]}$, the temperature $\theta=x^{(1+i) /[2+\sigma(1+i)]} T$ and the stream function $\psi=x^{(1+i) /[2+\sigma(1+i)]} f(\xi)$. Integrating once the resulting equations of momentum and energy with the boundary conditions $f=\theta_{\xi}=\left(\theta f_{\xi} / \xi^{i}\right)_{\xi}=0$ at $\xi=0$ reduce (2.2) and (2.3) to

$$
\xi^{i} \theta^{\sigma}\left(\frac{\theta f_{\xi}}{\xi^{i}}\right)_{\xi}+\frac{(1+i) f}{2+\sigma(1+i)}\left(\frac{\theta f_{\xi}}{\xi^{i}}\right)=0 \text { and } \xi^{i} \theta^{\sigma} \theta_{\xi}+\frac{(1+i) \operatorname{Pr} f}{2+\sigma(1+i)} \theta=0 .
$$



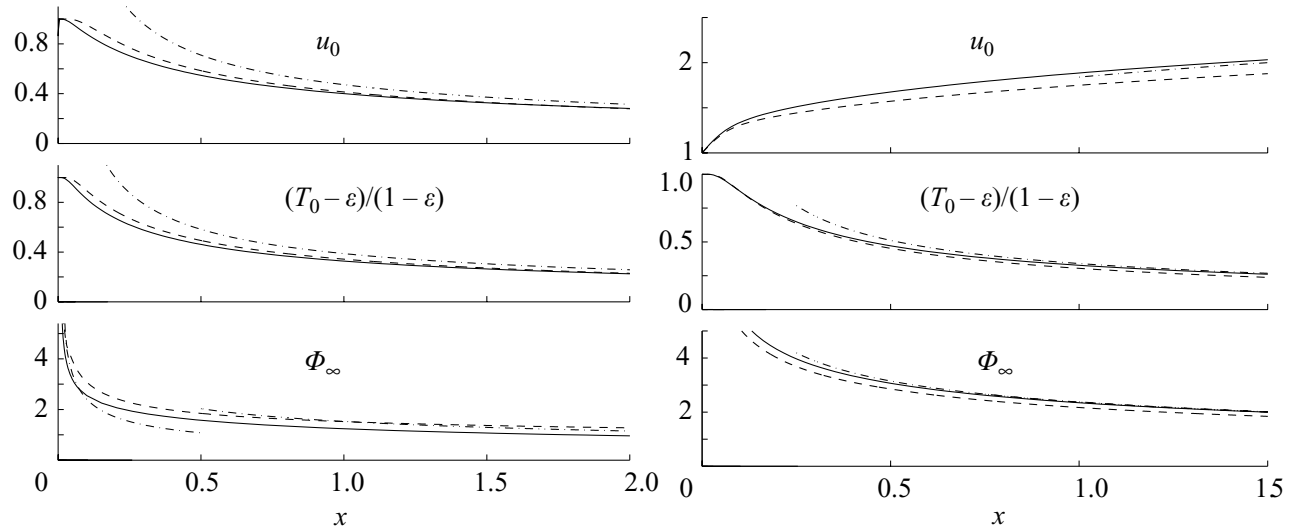

FIGURE 3. The variation of $u_{0},\left(T_{0}-\varepsilon\right) /(1-\varepsilon)$ and $\Phi_{\infty}$ for $\operatorname{Pr}=0.7, \sigma=0.7$ and $u_{c}=0$ for the axysimmetric jet with $G=0$ (left-hand-side plots) and $G=5$ (right-hand-side plots) as obtained by integration of (2.1)-(2.3) with $\varepsilon=0$ (solid lines) and $\varepsilon=0.1$ (dashed lines). The dot-dashed lines represent the asymptotic predictions for $x \gg 1$.
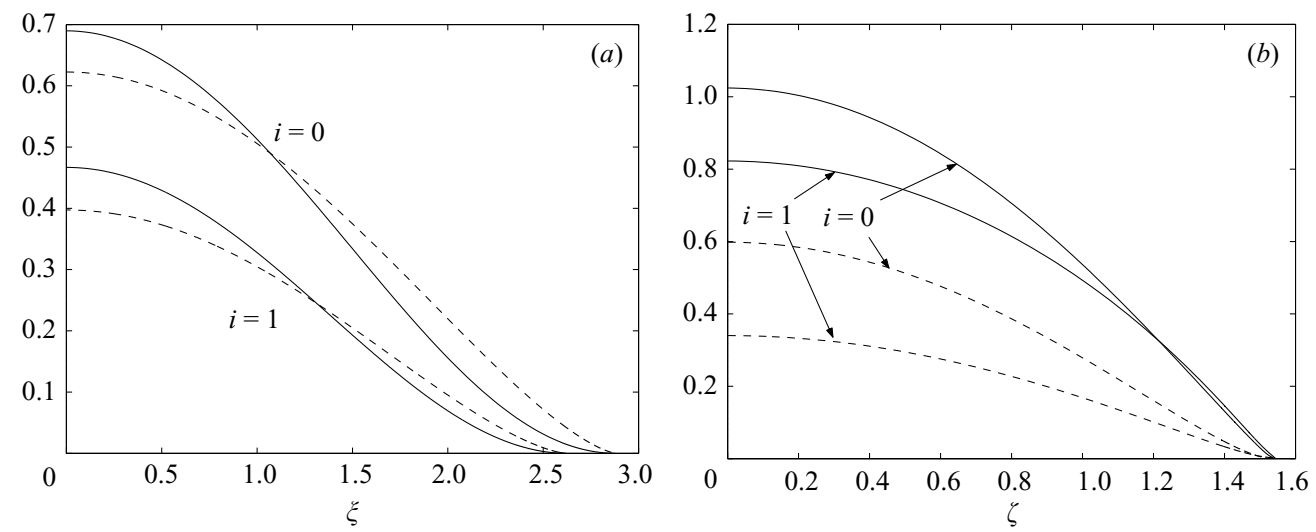

FIGURE 4. The self-similar temperature profile (dashed lines) and velocity profile (solid lines) obtained with $\operatorname{Pr}=0.7$ and $\sigma=0.7$ for the buoyancy-free jet $(a)$ and for the plume $(b)$.

The integration was started near the jet edge, where $\theta \simeq\left\{\left[(1+i) \sigma \operatorname{Pr} f_{f}\left(\xi_{f}-\xi\right)\right] /\right.$ $\left.\left[(2+\sigma(1+i)) \xi_{f}^{i}\right]\right\}^{1 / \sigma}$ and $\left.f \simeq f_{f}-C\left(\xi_{f}-\xi\right)^{[1-(1-\sigma)} \operatorname{Pr}\right] /(\sigma \operatorname{Pr})$. The values of the integration constants, $\xi_{f}=(2.856,2.707), f_{f}=(0.8121,7.036)$, and $C=(0.2658,2.614)$ for $i=(0,1)$, were selected in the iterative shooting scheme to satisfy the constraints $\int_{0}^{\xi_{f}} 2^{i} f_{\xi}^{2} \theta / \xi^{i} \mathrm{~d} \xi=\int_{0}^{\xi_{f}} 2^{i} f_{\xi} \theta \mathrm{d} \xi=1$ together with the regularity condition at the axis, giving the profiles of $\theta(\xi)$ and velocity $U(\xi)=x^{(1+i) /[2+\sigma(1+i)]} u=\theta f_{\xi} / \xi^{i}$ shown in figure $4(a)$, where the peak values are $\theta(0)=(0.623,0.398)$ and $U(0)=(0.691,0.467)$ for $i=(0,1)$. The solution provides in particular predictions for the jet entrainment rate $\Phi_{\infty}=x^{[i-1-\sigma(1+i)] /[2+\sigma(1+i)]}(1+i) f_{f} /[2+\sigma(1+i)]$ and for the jet boundary evolution $r_{f}=\xi_{f} x^{1 /[2+\sigma(1+i)]}$.

The accuracy of the far-field solution is tested in figure 2, where the profiles of temperature and velocity at $x=2.5$ and the prediction for the jet boundary, $r_{f}(x)$, are compared with those obtained numerically. Similarly, the asymptotic prediction for the jet entrainment rate is compared with the numerical results in figure 3 , giving good agreement over the range of $x$ considered. 
For $G \neq 0$, the buoyancy force, acting uniformly across the jet, continuously accelerates the hot gas, so that the solution for the jet in the far field becomes independent of the initial momentum flux. The balance among acceleration, shear stresses and buoyancy forces in (2.2) together with the condition of constant enthalpy flux (2.9) determine the characteristic values of the temperature, velocity and radius, suggesting the rescaled variables $\bar{\theta}=G^{(1-i) A} x^{(3+i) A} T, \bar{f}=G^{(i-1) A} x^{-(3+i) A} \psi$, and $\zeta=G^{(1+\sigma) A} x^{\sigma A} r$, where $A=[2 \sigma(1+i)+3+i]^{-1}$. The problem reduces to that of integrating

$$
\begin{aligned}
\left(\zeta^{i} \bar{\theta}^{\sigma} \bar{\theta}_{\zeta}\right)_{\zeta}+A(3+i) \operatorname{Pr}(\bar{f} \bar{\theta})_{\zeta} & =0 \\
{\left[\zeta^{i} \bar{\theta}^{\sigma}\left(\bar{\theta} \bar{f}_{\zeta} / \zeta^{i}\right)_{\zeta}\right]_{\zeta}+A(3+i) \bar{f}\left(\bar{\theta} \bar{f}_{\zeta} / \zeta^{i}\right)_{\zeta}-A \sigma(1+i) \bar{\theta} \bar{f}_{\zeta}^{2} / \zeta^{i}+\zeta^{i} } & =0
\end{aligned}
$$

with boundary conditions $\bar{\theta}_{\zeta}=\left(\bar{\theta} \bar{f}_{\zeta} / \zeta^{i}\right)_{\zeta}=\bar{f}=0$ at $\zeta=0$ and $\bar{\theta}=\bar{\theta} \bar{f}_{\zeta} / \zeta^{i}=0$ at $\zeta=\zeta_{f}$ and subject to the integral constraint $\int_{0}^{\zeta_{f}} 2^{i} \bar{\theta} \bar{f}_{\zeta} \mathrm{d} \zeta=1$. The corresponding profiles of temperature and velocity $\bar{U}=u /\left(G^{A(1+\sigma)(1+i)} x^{\sigma A(1+i)}\right)=\bar{\theta} \bar{f}_{\zeta} / \zeta^{i}$ are displayed in figure $4(b)$. The solution determines in particular the values of the jet boundary $\zeta_{f}=(1.545,1.546)$, maximum velocity $\bar{U}(0)=(1.024,0.8226)$, maximum temperature $\bar{\theta}(0)=(0.5981,0.3402)$, and stream function at the boundary $\bar{f}_{f}=(3.3150,4.028)$ for $i=(0,1)$. The latter can be used to compute the far-field entrainment rate $\Phi_{\infty}(x)=A(3+i) \bar{f}_{f} G^{A(1-i)} x^{A(3+i)-1}$, which is compared in figure 3 with results of numerical integrations. This figure also includes the far-field predictions for the velocity and temperature along the axis. Furthermore, the asymptotic profiles of temperature and velocity, plotted in figure 2 at $x=2.5$, and the prediction for the jet boundary $r_{f}=\zeta_{f} G^{-A(1+\sigma)} x^{-\sigma A}$, are practically indistinguishable from the numerical results with $\varepsilon=0$.

Note that the front character of the solution is lost when the temperature in the jet decays to values of the order of the ambient temperature, which occurs at distances of order $x \sim \varepsilon^{-[2+\sigma(1+i)] /(1+i)}$ for the buoyancy-free jet and at distances of order $x \sim G^{(i-1) /(3+i)} \varepsilon^{-1-2 \sigma(i+1) /(3+i)}$ for the plume. The description of this very far region would necessitate integration of the original equations (2.1)-(2.3) written for appropriately rescaled variables of order unity. The self-similar solutions identified above at $x \gg 1$ should be used as initial conditions in the integration.

\section{Hot jet in cold coflow}

In the presence of coflow, the boundary of the jet behaves as a contact surface separating the low-density gas from the cold coflow, with the radial velocity component $v_{f}(x)$ at $r=r_{f}$ satisfying $v_{f}(x)=u_{c}\left(\mathrm{~d} r_{f} / \mathrm{d} x\right)$. As before, one can use the similarity coordinate $\eta=(r-1) / \sqrt{x}$ and the stream function $\psi=\sqrt{x} F(\eta)$ to describe the thin annular mixing layer that forms at $x \ll 1$. The problem reduces to that of integrating (3.1) with boundary conditions $F-\eta=T-1=0$ as $\eta \rightarrow-\infty$ and $T=T F_{\eta}-u_{c}=0$ at $\eta=\eta_{f}$. The solution for the temperature and axial velocity is given in figure 1 for $u_{c}=(0.5,1.0,1.5)$ in terms of $\eta_{f}-\eta$. As in the case of the stagnant atmosphere, the integration determines the value of $\eta_{f}$, yielding for instance $\eta_{f}=0.6231$ for $u_{c}=0.5$ and $\eta_{f}=-0.4435$ for $u_{c}=1.5$. Note that this value gives the initial evolution of the jet radius $r_{f}=1+\eta_{f} \sqrt{x}$, which is plotted in figure 5 , and its accompanying radial velocity $v_{f}=\eta_{f} u_{c} /(2 \sqrt{x})$.

The procedure to integrate (2.1)-(2.3) is analogous to that used in the absence of coflow. Results of integrations corresponding to a buoyancy-free jet with $u_{c}=(0.5,1.5)$ are compared in figure 5 with results of integrations of the boundary-layer problem 

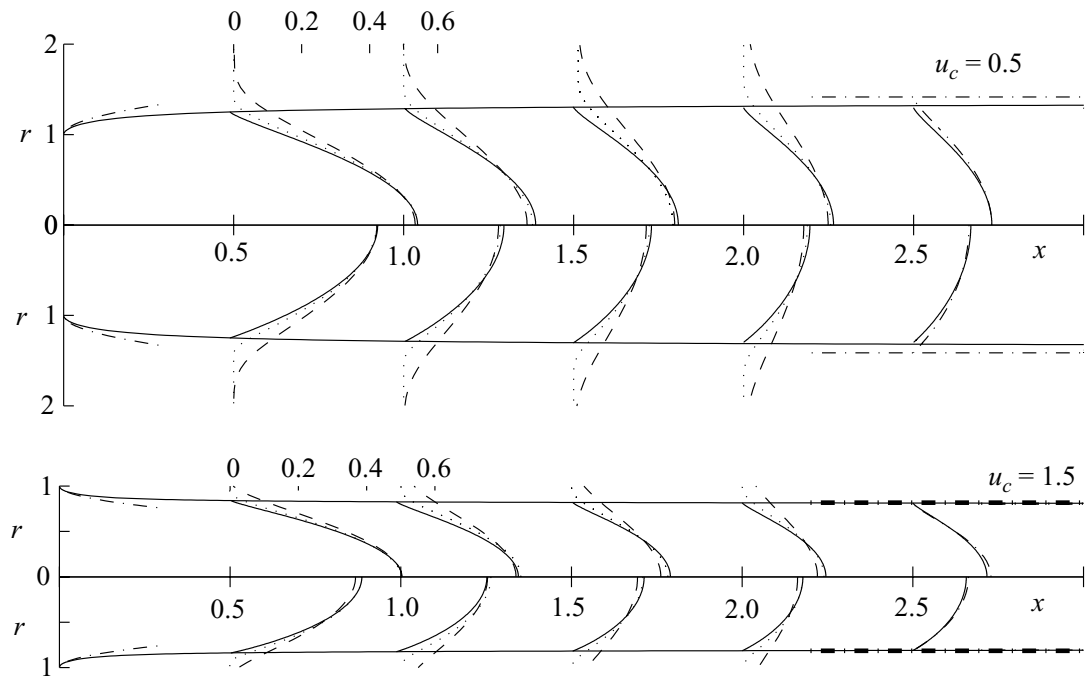

FIGURE 5. The profiles of velocity difference $\left(u-u_{c}\right) /\left(1-u_{c}\right)$ (upper half of the plots) and temperature difference $(T-\varepsilon) /(1-\varepsilon)$ (lower half of the plots) obtained for $\operatorname{Pr}=0.7, \sigma=0.7$, $G=0$ and $u_{c}=(0.5,1.5)$ by integration of (2.1)-(2.3) with $\varepsilon=0$ (solid line), $\varepsilon=0.02$ (dotted lines) and $\varepsilon=0.1$ (dashed lines). The scale for the velocity and temperature is indicated for the profiles at $x=0.5$ on the upper side of each plot. The profiles at $x=2.5$ are compared with the asymptotic solution for $x \gg 1$ (dot-dashed lines). The jet boundary corresponding to the front solution $\varepsilon=0$ is indicated as a solid line for completeness, and the asymptotic predictions $r_{f}=1+\eta_{f} \sqrt{x}$ for $x \ll 1$ and $r_{f}=1 / u_{c}^{1 /(1+i)}$ for $x \gg 1$ are included as dot-dashed lines.

(2.1)-(2.6) with $\varepsilon=(0.02,0.1)$, giving very good agreeement in the range of $x$ computed.

In the presence of gravity, the continuous acceleration of the hot gas leads to velocities that become much larger than $u_{c}$ for $x \gg 1$, so that the solution for the jet approaches that described above for the high-temperature plume. The far-field solution is different for the buoyancy-free jet considered in the figure, where the velocity differences from the coflow value become increasingly small for $x \gg 1$ and, consequently, the jet radius approaches the constant asymptotic value $r_{f}=1 / u_{c}^{1 /(i+1)}$, a result that follows from (2.9). In this far-field region, the radial velocity is given by $v=\operatorname{Pr}^{-1} T^{\sigma} \partial T / \partial r$, as can be seen from (2.3). Substituting this last result in (2.1) provides the nonlinear heat equation

$$
u_{c} \frac{\partial T}{\partial x}=\frac{T^{2}}{r^{i} P r} \frac{\partial}{\partial r}\left(T^{\sigma-1} r^{i} \frac{\partial T}{\partial r}\right) .
$$

Introducing the similarity variable $\Theta(R)=\left(\operatorname{Pr} u_{c}^{i-1} / x\right)^{-1 /(1+\sigma)} T$ in terms of the coordinate $R=r / r_{f}$ reduces (4.1) to $(\sigma+1)^{-1}+\left(\Theta / R^{i}\right)\left(R^{i} \Theta^{\sigma-1} \Theta_{R}\right)_{R}=0$, subject to the boundary conditions $\Theta_{R}(0)=\Theta(1)=0$. This canonical problem was encountered by Kurdyumov, Sánchez \& Liñán (2003) when investigating the long-time evolution of a symmetrical high-temperature hot spot in a gas. The integration for $\sigma=0.7$ give as peak values $\Theta(0)=0.4843$ for $i=0$ and $\Theta(0)=0.3450$ for $i=1$.

It is easy to verify from (2.2) that the accompanying velocity difference is simply given by $u-u_{c}=B\left(u_{c}^{i-1} \operatorname{Pr} / x\right)^{1 /(1+\sigma)} \Theta^{1 / P r}$ where the factor $B=\left(\int_{0}^{1} 2^{i} R^{i} \Theta^{1 / P r-1} \mathrm{~d} R\right)^{-1}$ was calculated from the integral condition $\int_{0}^{r_{f}} 2^{i} r^{i} \rho u\left(u-u_{c}\right) \mathrm{d} r=1-u_{c}$, yielding $B=1.698$ for $i=0$ and $B=2.2163$ for $i=1$. This far-field solution is tested in figure 5, 
where the axial velocity and temperature profiles obtained numerically at $x=2.5$ for $\varepsilon=0$ are compared with the asymptotic predictions, giving excellent agreement.

\section{Conclusions}

We have used the limit of small ambient-to-jet temperature ratios $\varepsilon \ll 1$ to describe the development region of high-temperature laminar gas jets, accounting for the temperature variation of viscosity and heat conductivity. The front descriptions appearing at leading order are seen to provide a very accurate representation for the temperature and velocity in the near-axis region of round and planar jets with moderately small values of $\varepsilon$. The applicability of these fronts, including their similarity far-field forms, to turbulent hot jets should be investigated in future work, which should also address the description of swirling jets, often encountered in applications. Our work is of interest for stability analyses of hot jets, such as that performed by Kennedy \& Chen (1998), providing the base profiles of temperature and velocity necessary in the stability development. Furthermore, fronts can also be expected to appear in vigorously exothermic jet diffusion flames, when the flame is embedded in an annular front region of very hot gas separating the inner fuel jet from the outer air.

This collaborative research was supported by the Spanish MEC under Projects\# ENE2005-08580-C02-01 and ENE2005-09190-C04-01.

\section{REFERENCES}

Anderson, D. A., Tannehill, J. C. \& Pletcher, R. H. 1984 Computational Fluid Mechanics and Heat Transfer. Hemisphere.

Bansal, J. L. \& Tack, S. S. 1978 Laminar plane jet in a gas medium. Z. Angew. Math. Phys. 29, $742-756$.

Bickley, W. 1937 The plane jet. Phil. Mag. 23, 727-731.

Bobnev, A. A. 1982 Exact solution for a high-temperature jet. J. Appl. Mech. Tech. Phys. (Historical Archive) 23, 647-651.

Bobnev, A. A. 1985 Class of self-similar solutions for a high-temperature axisymmetric jet. J. Appl. Mech. Tech. Phys. (Historical Archive) 26, 490-496.

Bobnev, A. A. 1986 Class of self-similar solutions for high-temperature plane and fan jets. High. Temp. 24, 212-219.

Crane, L. J. \& Pack, D. C. 1957 The laminar and turbulent mixing of jets of compressible fluid. Part 1. Flow far from the orifice. J. Fluid Mech. 2, 449-455.

Kennedy, C. A. \& Chen, J. H. 1998 Mean flow effects on the linear stability of compressible planar jets. Phys. Fluids 10, 615-626.

Kurdyumov, V., SÁnchez, A. L. \& LiÑ́n, A. 2003 Heat propagation from an external energy source in a gas. J. Fluid Mech. 491, 379-410.

PAI, S. I. 1949 Two-dimensional jet mixing of a compressible fluid. J. Aeronaut. Sci. 16, 463-469.

PAI, S. I. 1952 Axially symmetrical jet mixing of a compressible fluid. Q. Appl. Maths 10, 141-148.

PAI, S. I. 1954 Fluid Dynamics of Jets. D. Van Nostrand.

Schlichting, H. 1933 Laminare strahlausbreitung. Z. Angev. Math. Mech. 13, 260-263.

YIH, C. S. 1950 Temperature distribution in a steady laminar preheated air jet. J. Appl. Mech. 17, 381-382.

Zeldovich, Ya. B. \& Kompaneetz, A. S. 1950 Towards a theory of heat conduction with thermal conductivity depending on the temperature. Collection of papers dedicated to 70th birthday of Academician A. F. Ioffe, Izd. Akad. Nauk SSSR, Moscow, pp. 61-71. 